PROCEEDINGS OF THE

AMERICAN MATHEMATICAL SOCIETY

Volume 128, Number 3, Pages 857-864

S 0002-9939(99)05226-0

Article electronically published on July 28, 1999

\title{
ON CARATHÉODORY COMPLETENESS OF PSEUDOCONVEX REINHARDT DOMAINS
}

\author{
WŁODZIMIERZ ZWONEK
}

(Communicated by Steven R. Bell)

\begin{abstract}
We give a complete characterization of Carathéodory complete pseudoconvex Reinhardt domains, which extends results of Pflug, Fu and the author.
\end{abstract}

It is known that all bounded complete pseudoconvex Reinhardt domains are Carathéodory complete (see $[\mathrm{Pfl}]$ ). It was $\mathrm{S}$. Fu who extended this result to all bounded Reinhardt pseudoconvex domains satisfying some geometric condition (see $[\mathrm{Fu}]$ ) indicating that there are domains not satisfying this condition and not being Carathéodory complete. We prove that, actually, each pseudoconvex Reinhardt domain which is Carathéodory complete must fulfill the geometric condition from $[\mathrm{Fu}]$. Moreover, we do not need to restrict ourselves only to bounded domains but we extend the characterization to all Carathéodory hyperbolic pseudoconvex Reinhardt domains, making use of the characterization of such domains from [Zwo], where the characterization of the Kobayashi completeness of the domains under consideration was also given. This altogether gives a complete solution to the problem of characterization of the completeness of pseudoconvex Reinhardt domains with respect to invariant distances.

Before we state the main results of the paper let us recall the notation necessary for their formulation.

Let $D$ be a domain in $\mathbb{C}^{n}$, let $E$ be the unit disk in $\mathbb{C}$ and let $p$ denote the Poincaré distance on $E$. For points $w, z \in D$ we define

$$
\begin{gathered}
c_{D}(w, z):=\sup \{p(f(w), f(z)): f \in \mathcal{O}(D, E)\} ; \\
\tilde{k}_{D}(w, z):=\inf \left\{p\left(\lambda_{1}, \lambda_{2}\right): \text { there is } f \in \mathcal{O}(E, D) \text { with } f\left(\lambda_{1}\right)=w, f\left(\lambda_{2}\right)=z\right\} ; \\
k_{D}:=\text { the largest pseudodistance not larger than } \tilde{k}_{D} .
\end{gathered}
$$

We call $c_{D}$ (respectively, $k_{D}$ ) the Carathéodory (respectively, Kobayashi) pseudodistance. $\tilde{k}_{D}$ is called the Lempert function. Following [Jar-Pfl] we introduce the following notation:

$$
d_{D}^{*}:=\tanh d_{D}
$$

where $d$ denotes $c, k$ or $\tilde{k}$.

Received by the editors May 12, 1998.

1991 Mathematics Subject Classification. Primary 32H15; Secondary 32H20, 32F05.

The author is a fellow of the Alexander von Humboldt Foundation.

(C)1999 American Mathematical Society 
For $w, z \in D$ we also define the pluricomplex Green function:

$$
g_{D}(w, z):=\sup \{u(z)\}
$$

where the supremum is taken over all $u \in \operatorname{PSH}(D), u<0$ and such that $u(\cdot)-$ $\log \|\cdot-w\|$ is bounded from above near $w$.

Below we recall some properties of the above defined functions that we shall use in the sequel (see e.g. [Kob], [Kli], [Jar-Pfl]).

The following inequalities hold:

$$
c_{D} \leq k_{D} \leq \tilde{k}_{D}, c_{D}^{*} \leq \exp g_{D} \leq \tilde{k}_{D}^{*} .
$$

Moreover, $g_{D}(w, \cdot)$ is the largest plurisubharmonic function not exceeding the function $\log \tilde{k}_{D}^{*}(w, \cdot)$.

For a holomorphic mapping $F: D \mapsto G$ the following contracting property holds:

$$
d_{G}(F(w), F(z)) \leq d_{D}(w, z), w, z \in D .
$$

In particular, if $F$ is biholomorphic, then we have the above equality. For an increasing sequence of domains $\left\{D_{j}\right\}_{j=1}^{\infty}$ such that $\bigcup_{j=1}^{\infty} D_{j}=D$ we have that $d_{D_{j}}$ tends decreasingly to $d_{D}$ ( $d$ denotes any of the functions defined above).

Let $F: D \mapsto G$ be a holomorphic covering, $w, z \in G, \tilde{w} \in D$ and $F(\tilde{w})=w$. Then

$$
\tilde{k}_{G}(w, z)=\inf \left\{\tilde{k}_{D}(\tilde{w}, \tilde{z})\right\}, k_{G}(w, z)=\inf \left\{k_{D}(\tilde{w}, \tilde{z})\right\},
$$

where the infimum is taken over all $\tilde{z} \in D$ such that $F(\tilde{z})=z$.

The functions $k_{D}$ and $c_{D}$ are continuous whereas $\tilde{k}_{D}$ and $g_{D}$ are only upper semicontinuous.

We say that a domain $D$ is d-hyperbolic $(d=c, k$ or $\tilde{k})$ if $d_{D}(w, z)>0$ whenever $w \neq z$. We say that a domain $D$ is Brody hyperbolic if every holomorphic mapping $f: \mathbb{C} \mapsto D$ is constant.

It is trivial that for a domain $D$ the following chain of implications holds:

$$
c \text {-hyperbolic } \Longrightarrow k \text {-hyperbolic } \Longrightarrow \tilde{k} \text {-hyperbolic } \Longrightarrow \text { Brody hyperbolic. }
$$

In the case when the above defined functions are distances it is natural to introduce the notion of completeness. More precisely, assume that $D$ is $d$-hyperbolic $(d=c$ or $k$ ); then we say that a domain $D$ is $d$-complete if any $d_{D}$-Cauchy sequence $\left\{z^{\nu}\right\}_{\nu=1}^{\infty}$ is convergent to some $z^{0} \in D$ with respect to the standard topology in $D$.

A notion of finite compactness, closely related to completeness, may also be introduced. Namely, a $d$-hyperbolic domain $D$ is $d$-finitely compact if any $d_{D}$-ball with center in $D$ is a relatively compact subset of $D$ ( $d$ equals $c$ or $k$ ).

A domain $D \subset \mathbb{C}^{n}$ is called Reinhardt if $\left(\lambda_{1} z_{1}, \ldots, \lambda_{n} z_{n}\right) \in D$ for all points $z=\left(z_{1}, \ldots, z_{n}\right) \in D$ and $\left|\lambda_{1}\right|=\ldots=\left|\lambda_{n}\right|=1$.

Let us denote

$$
V_{j}:=\left\{z \in \mathbb{C}^{n}: z_{j}=0\right\}, j=1, \ldots, n .
$$

For $\alpha \in \mathbb{Z}^{n}$ we define $z^{\alpha}:=z_{1}^{\alpha_{1}} \cdot \ldots \cdot z_{n}^{\alpha_{n}}$ (for those $z$ for which it makes sense). Let us consider matrices $A:=\left(A_{k}^{j}\right)_{j=1, \ldots, n, k=1, \ldots, n} \in \mathbb{Z}^{n \times n}$ with $\operatorname{rank} A=n$.

For $z=\left(z_{1}, \ldots, z_{n}\right) \in \mathbb{C}^{n}$ for which it makes sense we define:

$$
\Phi_{A}(z):=z^{A}:=\left(z^{A^{1}}, \ldots, z^{A^{n}}\right),
$$

where $A^{j}:=\left(A_{1}^{j}, \ldots, A_{n}^{j}\right) \in \mathbb{Z}^{n}, j=1, \ldots, n$. 
Define $\mathbb{C}_{*}^{n}:=\left(\mathbb{C}_{*}\right)^{n}, \mathbb{C}_{*}:=\mathbb{C} \backslash\{0\}$. For all $A \in \mathbb{Z}^{m \times n}$ the mapping $\Phi_{A} \in$ $\mathcal{O}\left(\mathbb{C}_{*}^{n}, \mathbb{C}_{*}^{n}\right)$. It is easy to see that $\Phi_{A}$ is a biholomorphism of $\mathbb{C}_{*}^{n}$ iff $|\operatorname{det} A|=1$; in that case $\Phi_{A}^{-1}=\Phi_{A^{-1}}$.

Two Reinhardt domains $D$ and $G$ are called algebraically equivalent if there is a mapping $\Phi_{A}$ mapping $D$ biholomorphically onto $G$.

The following theorem giving a description of different notions of hyperbolicity and Kobayashi completeness in the class of Reinhardt pseudoconvex domains may be found in [Zwo].

Theorem 1 (see $[\mathrm{Zwo}]$ ). Assume that $D$ is a Reinhardt pseudoconvex domain in $\mathbb{C}^{n}$. Then the following conditions are equivalent:

(i) $D$ is c-hyperbolic;

(ii) $D$ is $\tilde{k}$-hyperbolic;

(iii) $D$ is Brody hyperbolic;

(iv) $D$ is algebraically biholomorphic to a bounded Reinhardt domain;

(v) $D$ is k-complete.

It follows from Theorem 1 that different notions of hyperbolicity coincide in the class of pseudoconvex Reinhardt domains, so the notion hyperbolic without any prefix is well-defined in the given class of domains.

Our aim is to deal with the problem of $c$-completeness. In view of Theorem 1 this problem concerns the same class of domains (i.e., hyperbolic domains). Our aim is the following theorem:

Theorem 2. Let $D$ be a hyperbolic pseudoconvex Reinhardt domain. Then the following conditions are equivalent:

(i) $D$ is c-finitely compact;

(ii) $D$ is c-complete;

(iii) $D$ is bounded and for any $j \in\{1, \ldots, n\}$

$$
\text { if } \bar{D} \cap V_{j} \neq \emptyset \text {, then } D \cap V_{j} \neq \emptyset \text {. }
$$

The geometric condition (3) is, as mentioned earlier, to be found in [Fu], where the implication (iii) $\Longrightarrow$ (i) is proved with methods from [Pfl]. It is also worth mentioning that the notions of $c$-completeness and $c$-finite compactness coincide on domains in $\mathbb{C}$; it is, however, not known whether the same remains true in higher dimension (for details see e.g. [Jar-Pfl]).

Before we start the proof let us present two simple lemmas:

Lemma 3. Let $D \subset \mathbb{C}_{*}^{n}$ be a pseudoconvex Reinhardt domain. Then $\tilde{k}_{D}=k_{D}$. In particular, $\tilde{k}_{D}$ is continuous.

Proof of Lemma 3. Let $\Omega:=\log D+i \mathbb{R}^{n}$, where $\log D:=\left\{x \in \mathbb{R}^{n}:\left(e^{x_{1}}, \ldots, e^{x_{n}}\right) \in\right.$ $D\}$. We know that $\log D$ is convex, therefore $\Omega$ is a convex tube domain and, consequently, we have that $\tilde{k}_{\Omega}=k_{\Omega}$ (see [Lem]). But the mapping

$$
\Omega \ni z \mapsto\left(\exp \left(z_{1}\right), \ldots, \exp \left(z_{n}\right)\right) \in D
$$

is a holomorphic covering, so in view of (2) we finish the proof.

The next lemma is a special case of the extended maximum principle (see e.g. [Hay-Ken], Theorem 5.16), formulated in the form that we shall need in the proof of Theorem 2: 
Lemma 4. Let $H_{0}:=\{\operatorname{Re} \lambda<0\}, u \in \mathrm{SH}\left(H_{0}\right)$ and $u<0$. Assume that for some $-\infty<a<0$ there is $M<0$ such that

$$
u(\lambda) \leq M \text { for } \lambda \in H_{0} \text { with } \operatorname{Re} \lambda=a .
$$

Then $u(\lambda) \leq M$ for any $\lambda \in H_{0}, \operatorname{Re} \lambda<a$.

In the proof of Theorem 2 we shall need the following characterization:

Proposition 5. Let $D$ be a hyperbolic pseudoconvex Reinhardt domain. Then the following conditions are equivalent:

(i) $D$ is algebraically equivalent to an unbounded domain;

(ii) $D$ is algebraically equivalent to a bounded domain $\tilde{D}$ such that

$$
\text { there is } j \in\{1, \ldots, n\} \text { with } \overline{\tilde{D}} \cap V_{j} \neq \emptyset \text { and } \tilde{D} \cap V_{j}=\emptyset \text {. }
$$

Proof of Proposition 5. ((ii) $\Longrightarrow$ (i)). Note that the mapping

$$
\tilde{D} \ni z \mapsto\left(z_{1}, \ldots, \frac{1}{z_{j}}, \ldots, z_{n}\right) \in \mathbb{C}^{n}
$$

is well-defined on $\tilde{D}$ and maps $\tilde{D}$ biholomorphically onto an unbounded domain, which completes the proof of this implication.

$((\mathrm{i}) \Longrightarrow(\mathrm{ii}))$. Certainly, we may assume that $D$ is unbounded. Let $\Phi_{A}$ be a biholomorphism of a bounded domain $\tilde{D}$ onto $D$ (see Theorem 1(iv)). We shall show that the domain $\tilde{D}$ has the desired property. Since $\tilde{D}$ is bounded it is sufficient to show that there is $j \in\{1, \ldots, n\}$ such that

$$
\bar{D} \cap V_{j} \neq \emptyset \text { and } \tilde{D} \cap V_{j}=\emptyset \text {. }
$$

Suppose that it does not hold. Then without loss of generality we may assume that for some $k, 0 \leq k \leq n$ :

$$
\tilde{D} \cap V_{j} \neq \emptyset, j=1, \ldots, k \text {, and } \tilde{\tilde{D}} \cap V_{j}=\emptyset, j=k+1, \ldots, n .
$$

The above conditions imply that:

$$
A_{j}^{r} \geq 0, j=1, \ldots, k, r=1, \ldots, n,
$$

and there is $M>0$ such that, for any $z \in \tilde{D},\left|z_{j}\right| \geq M, j=k+1, \ldots, n$ (here we need also the boundedness of $\tilde{D})$.

This gives us that (remember that $\tilde{D}$ is bounded) $\left\|z^{A^{r}}\right\|_{\tilde{D}}<\infty, r=1, \ldots, n$, which gives us that $D$ is bounded - a contradiction.

Define $p_{j}(x):=x_{j}, x \in \mathbb{R}^{n}, j=1, \ldots, n$.

Lemma 6. Let $\Omega \subset \mathbb{R}^{n}$ be an unbounded convex domain. Assume that

$$
\sup p_{j}(\Omega)<\infty, j=1, \ldots, n .
$$

Then for any $a \in \Omega$ there are an open set $U$ with $a \in U$ and $v \in\left(\mathbb{R}_{-}\right)^{n} \backslash\{0\}$ such that $U+\mathbb{R}_{+} v \subset \Omega$.

Proof. Assume that $a=0$. Let $h$ be the Minkowski functional of $\Omega$. Then $h$ is a continuous function. From the assumptions of the lemma we get the existence of $\left\{x^{\nu}\right\}_{\nu=1}^{\infty} \subset \Omega$ such that for some $j$ we have $x_{j}^{\nu} \rightarrow-\infty$. From the definition of $h$ we have that $h\left(x^{\nu}\right)<1$. But the homogeneity of $h$ implies that $h\left(\frac{x^{\nu}}{\left\|x^{\nu}\right\|}\right)<\frac{1}{\left\|x^{\nu}\right\|}$. Choosing a subsequence we get the existence of $v \in S^{n-1}$ such that $h(v)=0$. This 
implies that $\mathbb{R}_{+} v \subset \Omega$. Because of (5) we have that $v \in\left(\mathbb{R}_{-}\right)^{n}$. Simple properties of convexity give the existence of the open set $U$ as desired in the lemma.

Proof of Theorem 2. The implication ((i) $\Longrightarrow$ (ii)) is trivial. The implication ((iii) $\Longrightarrow(\mathrm{i})$ ) follows from $[\mathrm{Fu}]$. Therefore, we are left only with the implication ((ii) $\Longrightarrow$ (iii)).

Suppose that (iii) does not hold. This implies, in view of Proposition 5, that we may assume, using if necessary an algebraic biholomorphism, that $D$ is a bounded domain such that, for some $j \in\{1, \ldots, n\}, \bar{D} \cap V_{j} \neq \emptyset$ and $D \cap V_{j}=\emptyset$.

Without loss of generality we may assume that there are $1 \leq k \leq l \leq n$ such that

$$
\begin{gathered}
\bar{D} \cap V_{j} \neq \emptyset \text { and } D \cap V_{j}=\emptyset, j=1, \ldots, k, \\
\bar{D} \cap V_{j}=\emptyset, j=k+1, \ldots, l, \\
D \cap V_{j} \neq \emptyset, j=l+1, \ldots, n .
\end{gathered}
$$

Our first aim is to show that we may reduce our considerations only to the case $l=n$. In fact, put $\tilde{D}:=D \cap V_{l+1} \cap \ldots \cap V_{n}$. Certainly, $\tilde{D}$ is $c$-finitely compact, too. Then, after identification, $\tilde{D} \subset \mathbb{C}^{l}, \tilde{D} \cap V_{j}=\emptyset, j=1, \ldots, l$, and $\tilde{\tilde{D}} \cap V_{j}=\emptyset, j=$ $k+1, \ldots, l$. Moreover, using the description of pseudoconvex Reinhardt domains (see [Vla]), one may easily verify that $\tilde{\tilde{D}} \cap V_{j} \neq \emptyset, j=1, \ldots, k$.

We assume that $D$ is bounded and

$$
D \subset \mathbb{C}_{*}^{n}, \bar{D} \cap V_{j} \neq \emptyset, j=1, \ldots, k, \bar{D} \cap V_{j}=\emptyset, j=k+1, \ldots, n,
$$

where $1 \leq k \leq n$.

Without loss of generality we may assume that $(1, \ldots, 1) \in D$. Applying Lemma 6 to the domain $\Omega:=\log D$ and the point $a:=(0, \ldots, 0)$ we get that there is a vector $v \in\left(\mathbb{R}_{-}\right)^{n} \backslash\{0\}$ and a neighborhood $U$ of $a$ such that

$$
x+t v \in \log D \text { for any } x \in U, t>0 .
$$

In view of (6) we lose no generality assuming that $v=\left(v_{1}, \ldots, v_{l}, 0, \ldots, 0\right)$, where $v_{j}<0$ and $1 \leq l \leq k$ ( $l$ fixed). Put $\alpha_{j}:=-v_{j}, j=1, \ldots, l$. We may also assume that $\alpha_{1}=1$. Then we have that

$$
\left(e^{x_{1}} \exp (t), e^{x_{2}} \exp \left(t \alpha_{2}\right), \ldots, e^{x_{l}} \exp \left(t \alpha_{l}\right), e^{x_{l+1}}, \ldots, e^{x_{n}}\right) \in D \text { for } t<0, x \in U .
$$

In particular,

$$
\left(\exp (\lambda), \mu_{2} \exp \left(\lambda \alpha_{2}\right), \mu_{3} \exp \left(\lambda \alpha_{3}\right), \ldots, \mu_{l} \exp \left(\lambda \alpha_{l}\right), 1, \ldots, 1\right) \in D
$$

for $\lambda \in H_{0}$ (where $H_{R}:=\{\operatorname{Re} \lambda<R\} \subset \mathbb{C}, 0 \leq R \leq \infty$ ), $\mu_{j} \in P:=\left\{e^{-\varepsilon}<|\mu|<\right.$ $\left.e^{\varepsilon}\right\} \subset \mathbb{C}, j=2, \ldots, l$, and $\varepsilon>0$ is suitably small.

For $\left(\lambda, \mu_{2}, \ldots, \mu_{l}\right) \in H_{R} \times P^{l-1}$ we define

$$
\Phi_{R}\left(\lambda, \mu_{2}, \ldots, \mu_{l}\right):=\left(\exp (\lambda), \mu_{2} \exp \left(\lambda \alpha_{2}\right), \mu_{3} \exp \left(\lambda \alpha_{3}\right), \ldots, \mu_{l} \exp \left(\lambda \alpha_{l}\right)\right) \in \mathbb{C}^{l} .
$$

Put $G_{R}:=\Phi_{R}\left(H_{R} \times P^{l-1}\right)$. We have $G_{R} \subset G_{R^{\prime}}$ if $R<R^{\prime}$ and $\bigcup_{R<\infty} G_{R}=G_{\infty}$.

Since $G_{R}$ is a pseudoconvex Reinhardt domain lying in $\mathbb{C}_{*}^{l}$ we know from Lemma 3 that $\tilde{k}_{G_{R}}$ is continuous (for $0 \leq R \leq \infty$ ).

Note that $G_{0} \times\{1\}^{n-l} \subset D$ and $(0, \ldots, 0,1, \ldots, 1) \notin D$; therefore, to complete the proof it is sufficient to find, for a given sequence $\left\{a_{\nu}\right\}_{\nu=1}^{\infty}$ with $a_{\nu}>0$ such that $\sum_{\nu=1}^{\infty} a_{\nu}<\infty$, a sequence $\left\{z^{\nu}\right\}_{\nu=1}^{\infty} \subset G_{0}$ such that $z_{1}^{\nu} \rightarrow 0$ and

$$
\log c_{G_{0}}^{*}\left(z^{\nu}, z^{\nu+1}\right) \leq g_{G_{0}}\left(z^{\nu}, z^{\nu+1}\right) \leq \log a_{\nu} .
$$


For $0 \leq R<\infty$ put

$$
\Psi_{R}: G_{0} \ni z \mapsto\left(\exp (R) z_{1}, \exp \left(\alpha_{2} R\right) z_{2}, \ldots, \exp \left(\alpha_{l} R\right) z_{l}\right) \in G_{R} .
$$

Note that $\Psi_{R}$ is a biholomorphism.

Define $\varphi_{R}(\lambda):=\Phi_{R}(\lambda, 1, \ldots, 1), \lambda \in H_{R}$. Note that

$$
\tilde{k}_{G_{\infty}}\left(\varphi_{\infty}(-1), \varphi_{\infty}(\lambda)\right)=0, \lambda \in \mathbb{C} .
$$

The continuity of $\tilde{k}_{G_{\infty}}$ implies that

$$
\tilde{k}_{G_{\infty}}\left(\varphi_{\infty}(-1), z\right)=0 \text { for any } z \in \overline{\varphi_{\infty}(\mathbb{C})}
$$

(the closure above is taken in $G_{\infty}$ ). Now Dini's Lemma implies that for any $\nu$ there is $R_{\nu}>0\left(\left\{R_{\nu}\right\}_{\nu=1}^{\infty}\right.$ may be assumed to be tending strictly increasingly to infinity) such that

$$
\tilde{k}_{G_{R_{\nu}}}^{*}\left(\varphi_{0}(-1), z\right)<a_{\nu} \text { for any } \exp (-2) \leq\left|z_{1}\right| \leq \exp (-1), z \in \overline{\varphi_{\infty}(\mathbb{C})},
$$

which implies that

$$
\tilde{k}_{G_{R_{\nu}}}^{*}\left(\varphi_{0}(-1), \varphi_{0}(\lambda)\right)<a_{\nu} \text { for any } \lambda \in \mathbb{C} \text { with }-2 \leq \operatorname{Re} \lambda \leq-1 .
$$

Applying the biholomorphism $\Psi_{R_{\nu}}^{-1}$ we get

$$
\tilde{k}_{G_{0}}^{*}\left(\varphi_{0}\left(-1-R_{\nu}\right), \varphi_{0}(\lambda)\right)<a_{\nu} \text { for any } \lambda \in \mathbb{C} \text { with }-2-R_{\nu} \leq \operatorname{Re} \lambda \leq-1-R_{\nu} .
$$

Now define $u(\lambda):=g_{G_{0}}\left(\varphi_{0}\left(-1-R_{\nu}\right), \varphi_{0}(\lambda)\right), \lambda \in H_{0}$. Certainly, $u \in \operatorname{SH}\left(H_{0}\right)$, $u<0$. In view of (7) we have $u(\lambda)<\log a_{\nu}$ for $-2-R_{\nu} \leq \operatorname{Re} \lambda \leq-1-R_{\nu}$, from which, in view of Lemma 4 , we conclude

$$
g_{G_{0}}\left(\varphi_{0}\left(-1-R_{\nu}\right), \varphi_{0}(\lambda)\right)=u(\lambda) \leq \log a_{\nu} \text { for any } \lambda \in \mathbb{C} \text { with } \operatorname{Re} \lambda<-1-R_{\nu} .
$$

To finish the proof it is sufficient to define

$$
z^{\nu}:=\varphi_{0}\left(-1-R_{\nu}\right)
$$

Remark 7. Remark that if there is $\alpha \in \mathbb{R} \cdot \mathbb{Q}_{+}^{l}(\alpha$ is from the proof of Theorem 2), e.g., when $l=1$, then without loss of generality we may assume that $\alpha \in \mathbb{Z}_{+}^{l}$ and the proof of Theorem 2 is much simpler. Actually, define $\varphi: E_{*} \mapsto D$ as

$$
\varphi(\lambda):=\left(\lambda^{\alpha_{1}}, \ldots, \lambda^{\alpha_{l}}, 1 \ldots, 1\right) .
$$

Take any $c_{E_{*}}$-Cauchy sequence $\left\{\lambda_{\nu}\right\}_{\nu=1}^{\infty}$ such that $\lambda_{\nu} \rightarrow 0$. Then we have

$$
g_{E_{*}}\left(\lambda_{\nu}, \lambda_{\mu}\right) \geq g_{D}\left(\varphi\left(\lambda_{\nu}\right), \varphi\left(\lambda_{\mu}\right)\right) \geq \log c_{D}^{*}\left(\varphi\left(\lambda_{\nu}\right), \varphi\left(\lambda_{\mu}\right)\right),
$$

from which we conclude the desired result.

Remark 8. From the proof of Theorem 2 we can conclude the following property: conditions (i)-(iii) are equivalent to the fact that

$$
\text { for any } z_{0} \in D, g_{D}\left(z_{0}, z\right) \rightarrow 0 \text { as } z \rightarrow \partial D \cup\{\infty\} \text {. }
$$

As simple conclusions from Theorems 1 and 2 we get a characterization of hyperconvex and taut Reinhardt domains.

First note that any taut domain is $\tilde{k}$-hyperbolic and any $k$-complete domain is taut (see [Jar-Pfl]); therefore, in view of Theorem 1 we get: 
Corollary 9. For a Reinhardt pseudoconvex domain the following conditions are equivalent

(i) $D$ is algebraically equivalent to a bounded domain;

(ii) $D$ is taut.

Let us define the hyperconvexity (see [Ste]) as follows: $D$ is hyperconvex if $D=$ $\{u<0\}$, where $u \in \operatorname{PSH}(D) \cap \mathcal{C}(D)$ and, for any $\varepsilon>0,\{u<-\varepsilon\} \subset \subset D$.

Note that in contrast to many authors we allow a hyperconvex domain to be unbounded, which causes the hyperconvex domains to be invariant with respect to biholomorphic mappings.

Corollary 10. Let $D$ be a Reinhardt pseudoconvex domain. Then the following conditions are equivalent:

(i) $D$ is bounded and for any $j \in\{1, \ldots, n\}$

$$
\text { if } \bar{D} \cap V_{j} \neq \emptyset \text {, then } D \cap V_{j} \neq \emptyset \text {; }
$$

(ii) $D$ is hyperconvex.

Proof. ((ii) $\Longrightarrow(\mathrm{i}))$. Let $D=\{u<0\}$, where $u$ is from definition of the hyperconvex domain. We first prove that $D$ is hyperbolic. If it were not the case, then in view of Theorem 1(iii) there would exist a non-constant holomorphic mapping $\varphi: \mathbb{C} \mapsto D$. Then $u_{\mid \varphi(\mathbb{C})} \equiv C$ for some $C<0$ but $\varphi(\mathbb{C})$ is not bounded, which contradicts the fact that $\{u<C / 2\}$ is relatively compact.

Since $g_{G}\left(z_{0}, z\right) \rightarrow 0$ as $z \rightarrow \partial G$ for any hyperconvex bounded domain $G$ and $z_{0} \in G$ (see e.g., [Kli] $)$, we complete the proof making use of Remark 8 .

$((\mathrm{i}) \Longrightarrow($ ii $))$. Fix $z_{0} \in D$. Put $u(z):=\log c_{D}^{*}\left(z_{0}, z\right), z \in D$. We know that $u \in \operatorname{PSH}(D) \cap \mathcal{C}(D)$ (see [Jar-Pfl]). On the other hand $c$-finite compactness of $D$ (see Theorem 2) implies that $\{u<-\varepsilon\} \subset \subset D$ for any $\varepsilon>0$.

The problem of characterizing bounded hyperconvex Reinhardt domains was also dealt with in [Car-Ceg-Wik].

Remark 11. It is worth mentioning that a simpler, direct proof of Corollary 10 was presented to the author by Professor P. Pflug. Namely, in view of the proof of Theorem 2, to prove Corollary 10 it is sufficient to disprove the hyperconvexity of the domain $G_{0}$ (as in the proof of Theorem 2). To do it we can proceed as follows. Let $u$ be an exhausting function from the definition of the hyperconvex domain. Define $v(z):=\sup \left\{u\left(z_{1} e^{i \theta_{1}}, \ldots, z_{l} e^{i \theta_{l}}\right), \theta_{j} \in \mathbb{R}\right\}$. It is easy to see that $v$ is an exhausting function from the definition of the hyperconvexity and, additionally, we have that $v(z)=v\left(\left|z_{1}\right|, \ldots,\left|z_{l}\right|\right)$. Therefore, the function $E_{*} \ni \lambda \mapsto v\left(|\lambda|,|\lambda|^{\alpha_{2}}, \ldots,|\lambda|^{\alpha_{l}}\right)$ is subharmonic and bounded from above by 0 ; therefore it can be continued subharmonically to $E$ but because of hyperconvexity the value at 0 would have to be 0 , which is possible only in case of the constant function - a contradiction.

\section{REFERENCES}

[Car-Ceg-Wik] M. Carlehed, U. Cegrell \& F. Wikström, Jensen Meauseres, Hyperconvexity and Boundary Behaviour of the Pluricomplex Green Function, Research Report No 15 Umea University (1997).

[Fu] S. Fu, On completeness of invariant metrics of Reinhardt domains, Arch. Math. 63 (1994), 166-172. MR 95h:32024

[Hay-Ken] W. K. Hayman \& P. B. Kennedy, Subharmonic Functions, volume 1, London Mathematical Society Monographs 9, Academic Press, London, 1976. MR 57:665 
[Jar-Pfl] M. Jarnicki \& P. Pflug, Invariant Distances and Metrics in Complex Analysis, Walter de Gruyter, 1993. MR 94k:32039

[Kli] M. Klimek, Pluripotential Theory, Oxford Science Publications, 1991. MR 93h:32021

[Kob] S. Kobayashi, Hyperbolic Manifolds and Holomorphic Mappings, Pure and Appl. Math. 2, M. Dekker, 1970. MR 43:3503

[Lem] L. Lempert, La metrique de Kobayashi et la representation des domaines sur la boule, Bull. Soc. Math. France 109 (1981), 427-474. MR 84d:32036

[Pfl $\quad$ P. Pflug, About the Carathéodory completeness of all Reinhardt domains, In: Functional Analysis, Holomorphy and Approximation Theory II, (1984), 331-337, G. Zapata ed. MR 86b:32026

[Ste] J. L. Stehlé, Fonctions plurisousharmoniques et convexité holomorphe de certaines fibrés analytiques. In, Séminaire Pierre Lelong (Analyse), Année 1973/1974 (ed. P. Lelong, P. Doulbeaut, H. Skoda) (1975), 155-179. MR 53:3368

[Vla] V. Vladimirov, Methods of the Theory of Functions of Several Complex Variables. (Russian) Moskau 1964. English transl., Cambridge, Mass.-London, 1966. MR 34:1551

[Zwo] W. Zwonek, On hyperbolicity of pseudoconvex Reinhardt domains, Arch. Math., to appear.

Instytut Matematyki, Reymonta 4, 30-059 Kraków, Poland

E-mail address: zwonek@im.uj.edu.pl

Current address: Carl von Ossietzky Universität Oldenburg, Fachbereich 6 - Mathematik, Postfach 2503, 26111 Oldenburg, Germany

E-mail address: zwonek@mathematik.uni-oldenburg.de 